\title{
Treatment of Class I Malocclusion with a Lower Incisor Extraction: A Cose Report
}

\author{
Dr Jitendra Kumar Sah,' Dr Praveen Mishra,,2 Dr Rabindra Man Shrestha,3 Dr Alka Gupta \\ 'PG Resident, ${ }^{2.3}$ Professor, ${ }^{4}$ Lecturer, Dept of Orthodontics, Kantipur Dental College, Kathmandu, Nepal \\ Correspondence: Dr Jitendra K Sah; Email: jkumarsah123@gmail.com
}

\section{ABSTRACT}

A case report is presented with Class I malocclusion, moderate lower anterior crowding, maxillary dental midline shift with missing right lateral incisor. The adult orthodontic case was treated comprehensively with the extractions of lower lateral incisor, and upper and lower left first premolars as the balancing extraction. Remarkable improvements were achieved in facial profile, axial anterior inclination, esthetic smile and midline correction in short treatment time. Thus, it is concluded that lower incisor extraction in moderate lower anterior crowding and midline shift yield better and stable results with minimal intervention in select cases.

Keywords: balancing extraction, interdigitation, intervention, rotation

\section{INTRODUCTION}

The main goal of orthodontic treatment is to obtain normal relationship of the teeth with facial structures. Angle emphasized that the preservation of all dental units was necessary to achieve facial balance, harmony and esthetics. However, subsequent studies related to the stability of results pointed the necessity of tooth extractions in order to correct certain malocclusions. The lower incisor inclination possesses a great importance in orthodontic diagnosis and treatment planning. Orthodontists realize that maintaining all dental units to correct malocclusions is not always possible. ${ }^{1-3}$ Extraction of selected permanent tooth is necessary in severe crowding cases. A great controversy exists between extraction and non extraction treatment protocol.

Orthodontic paradigm has shifted predominantly from non-extraction philosophy in the early 1900's to extraction-oriented regimen in middle of the twentieth century, and now again back to non-extraction emphasis. ${ }^{4}$ Several approaches for crowded mandibular anterior teeth are currently employed: distal movement of posterior teeth, lateral movement of canines, labial movement of incisors, interproximal enamel reduction, removal of premolars, removal of one or two incisors, and various combinations of the above. Selecting the best treatment is often difficult, and all guidelines do not apply to every case..$^{5}$ In 1757, Bourdet, a disciple of Pierre
Fauchard, recommended the removal of premolars to relieve crowding. Likewise, Hunter ${ }^{6}$ extracted the first premolars to allow incisor retrusion in cases of posterior protrusion. Almost two centuries later, $\mathrm{Hahn}^{7}$ advocated the removal of a mandibular incisor to close the space and thus, reduce anterior proclination.

Arch length deficiency is one of the critical factors in choosing either of the treatment protocols. ${ }^{8}$ Bolton analysis reveals the discrepancy between maxillary and mandibular dentitions. Deficiency in mesiodistal width of maxillary anterior teeth may provide an additional extraction option of removal of a mandibular incisor. Treatment using extraction of a single mandibular incisor is not popular in orthodontic practice. Previous articles by Valinoti, ${ }^{5}$ Shapiro 9 and Riedel ${ }^{10}$ have described the indications, advantages, and limitations of mandibular incisor extraction. Uribe and Nanda" ${ }^{11}$ clearly described the case selection and mechanics of treatment with the lower incisor extraction. According to Owen, ${ }^{4}$ patients who are suitable for single lower incisor extraction usually fit the following diagnostic pattern: Class I molar relationship, moderately crowded lower incisors, mild or no crowding in the upper arch, acceptable soft tissue profile, minimal to moderate overbite and overjet, no or minimal growth potential, and missing lateral incisors or peg shaped laterals. Unwanted side-effects are: increase of overbite and overjet beyond acceptable limits, space reopening, partly unsatisfactory posterior 
occlusion, recurrence of crowding in remaining incisors, and unaesthetic loss of interdental papillae in the mandibular anterior region. 5,6,10,12

\section{CASE REPORT}

A 29-year-old male reported to Department of Orthodontics, Kantipur Dental College, Kathmandu with the chief complains of irregularly placed teeth in lower front region. No relevant medical history was present. His upper right lateral incisor was extracted due to trauma at the age of ten years. Extraoral examination revealed leptoprosopic face with mesocephalic head. He had symmetric face with increased lower lip length. There were no signs of temporomandibular joint dysfunction and abnormal mandibular movement. Intraorally, upper dental midline was shifted towards right side by 3 millimeters. Lower anterior cortical plate was thin with thin and friable gingiva. There was inadequate incisal exposure during smile and lower incisors were exposed (Figure 1).

Study model showed bilateral Class I molar and canine relation with good posterior intercuspation. There was mild crowding in maxillary arch but mandibular arch had a crowding of $7 \mathrm{~mm}$; which was asymmetric and square shaped. Lower canines were blocked out into crossbite position. There was 50\% deepbite and Curve of Spee were $1.5 \mathrm{~mm}$ bilaterally.

Panoramic x-rays showed no anatomical and pathological abnormalities. Cephalometric analysis showed, maxilla and mandible were retropositioned with Class III skeletal pattern. Lower anterior face height was increased and growth vector was normal. Maxillary and mandibular dentitions were proclined and forwardly placed (Figure 2).

Lateral cervical vertebrae showed past peak growth. Etiology of malocclusion was unknown and assumed to be genetic. Perhaps the deepbite caused the mandibular crowding, or the collapse of the mandibular anterior dentition influenced the deep bite.

Treatment Objective: To relieve crowding, correct midline shift, correct axial inclination of teeth. Correcting malocclusion would also correct incisal display and improve smile aesthetics.

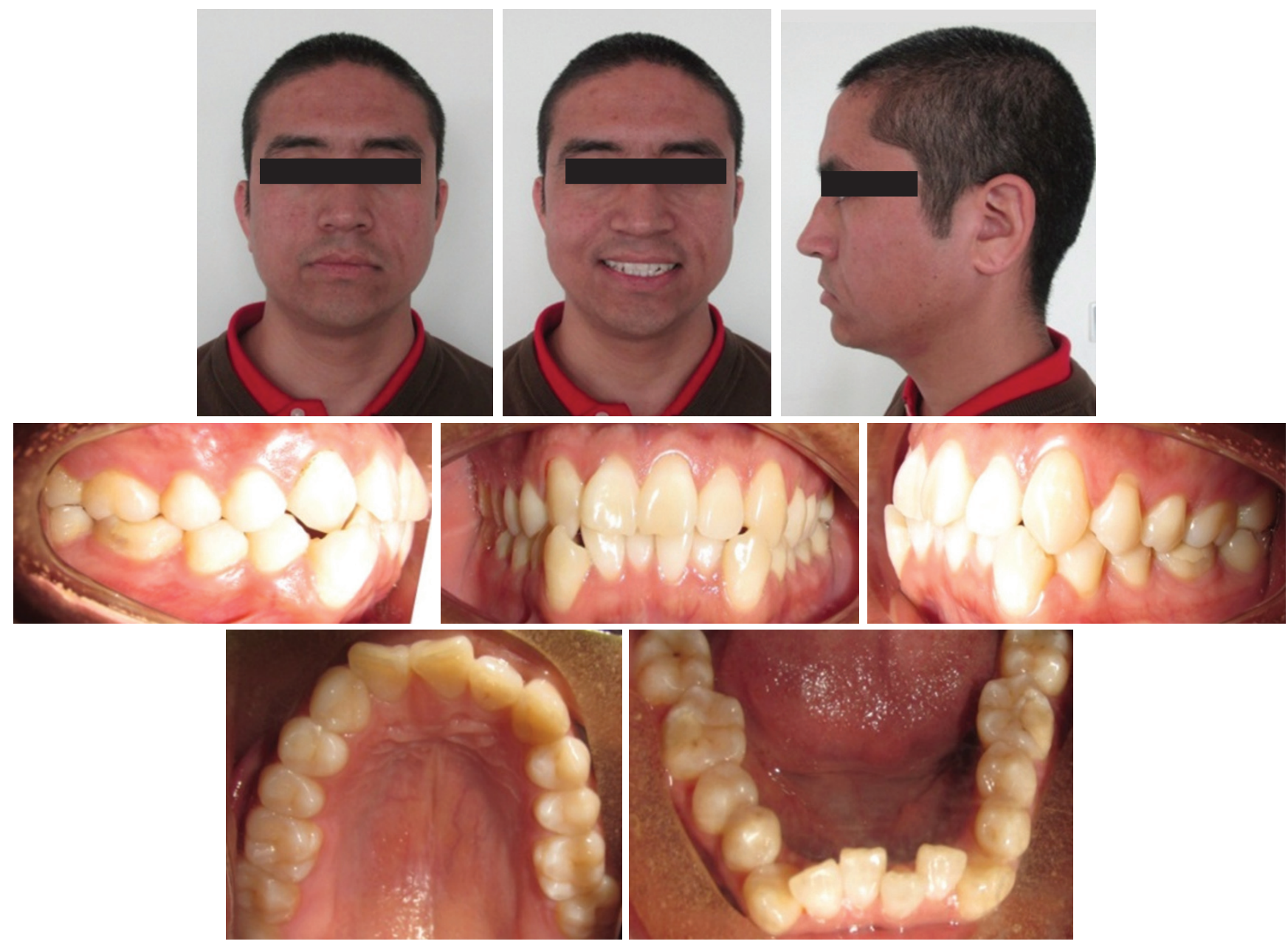

Figure 1: Pre-treatment intra-oral and extra-oral photographs 

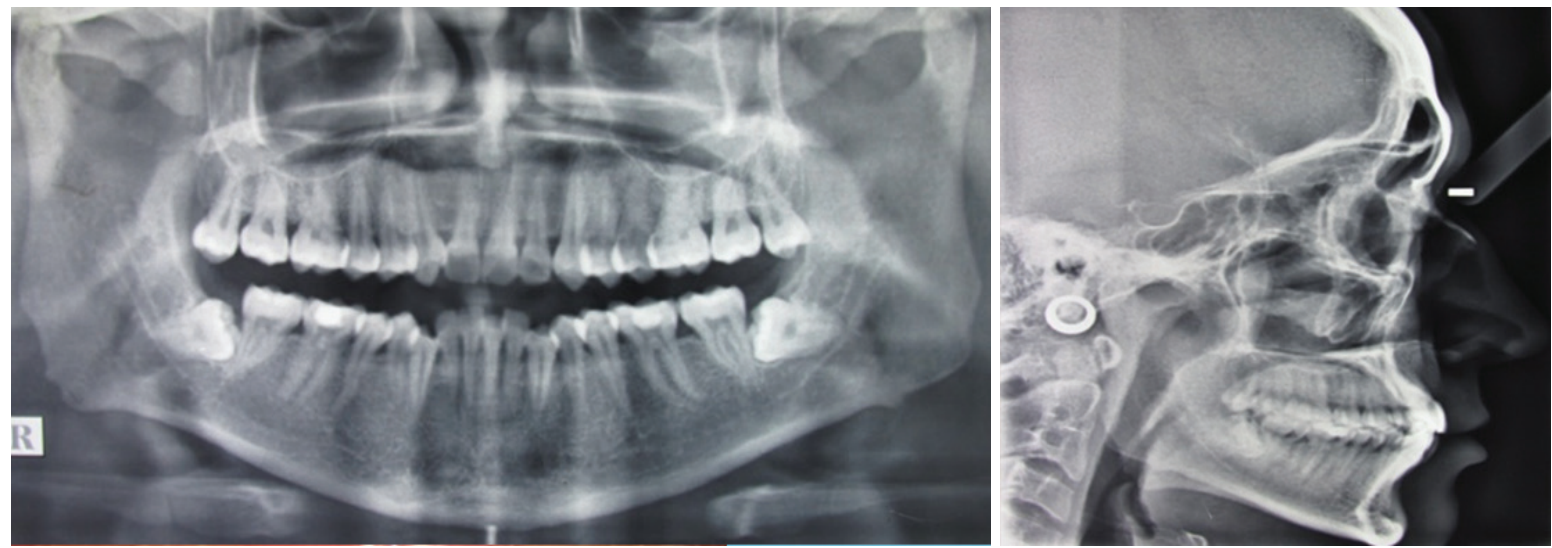

Figure 2: Pre-treatment OPG \& lateral cephalogram
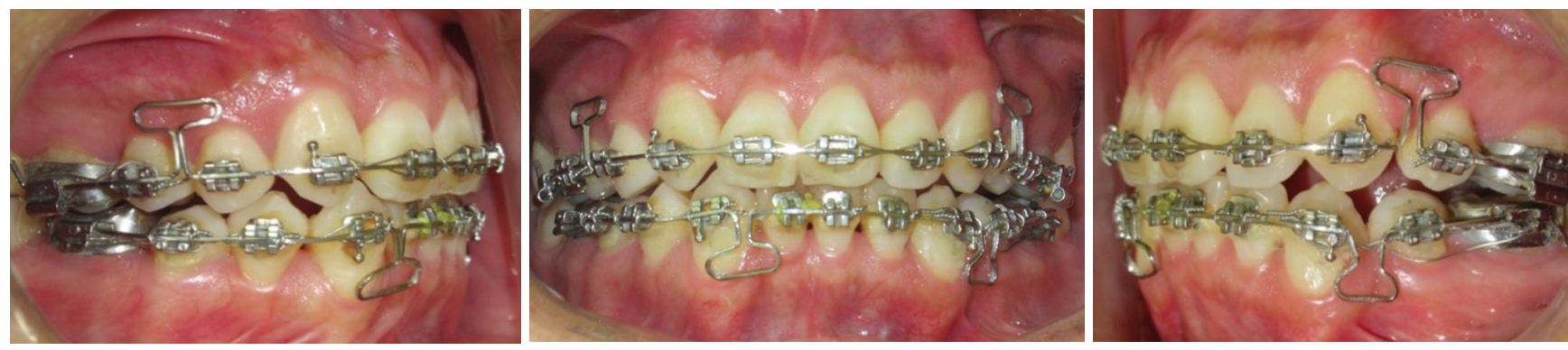

Figure 3: Mid-treatment intra-oral photographs

Treatment Alternatives: Many operators usually consider premolar extraction for leveling of arches and relieve lower incisor crowding. Due to thinning of lower anterior cortical plate, it was decided to extract 24, 34 and 42. Another option would be the creating space for 12 and rehabilitation with implant or fixed prosthesis. First alternative was deemed the best option.

Treatment progress: Moderate anchorage control was selected. Posterior bite plate was given for jaw disarticulation to correct crossbite. Fixed 0.018" Roth bracket was bonded in both arches. The wire progressed from 0.012", 0.014" Ni-Ti and 0.016" SS for leveling and alignment. E-thread ligation was used for lingual tipping of canines between the two bigger consolidated units. Leveling of arches was done with relative extrusion of posteriors. En mass retraction was done with $0.017 \times 0.025$ " using "T" loop (Figure 3). Compensatory bends were maintained in retraction wires. Class III elastics of 2.5 ounce were used during retraction.

Progress OPG revealed diverging roots in respect to canines and second premolars. 0.017 $\times 0.025$ " root paralleling wire incorporating three tear drop loops were activated in each quadrant (Figure 4).

During finishing, arches presented with Class II end-on left side and Class I on right side. Thus, Class III elastics on right and Class II on left side corrected molar relationship uneventfully. After 23 months of active treatment; wrap around retainers were prescribed in both arches for full time wear in first 9 months and following 6 months on partial time basis (Figure 5).
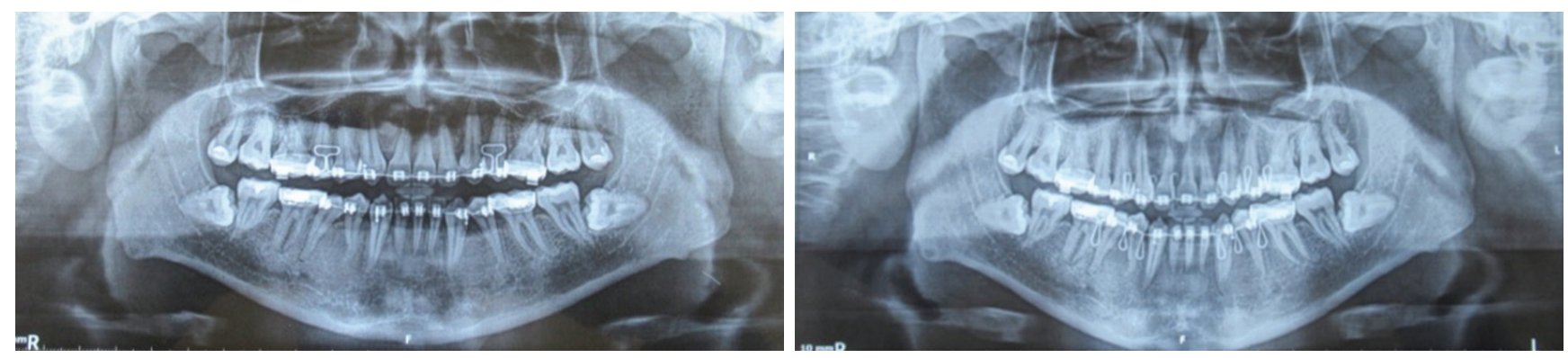

Figure 4: OPG's showing space closure and root paralleling 

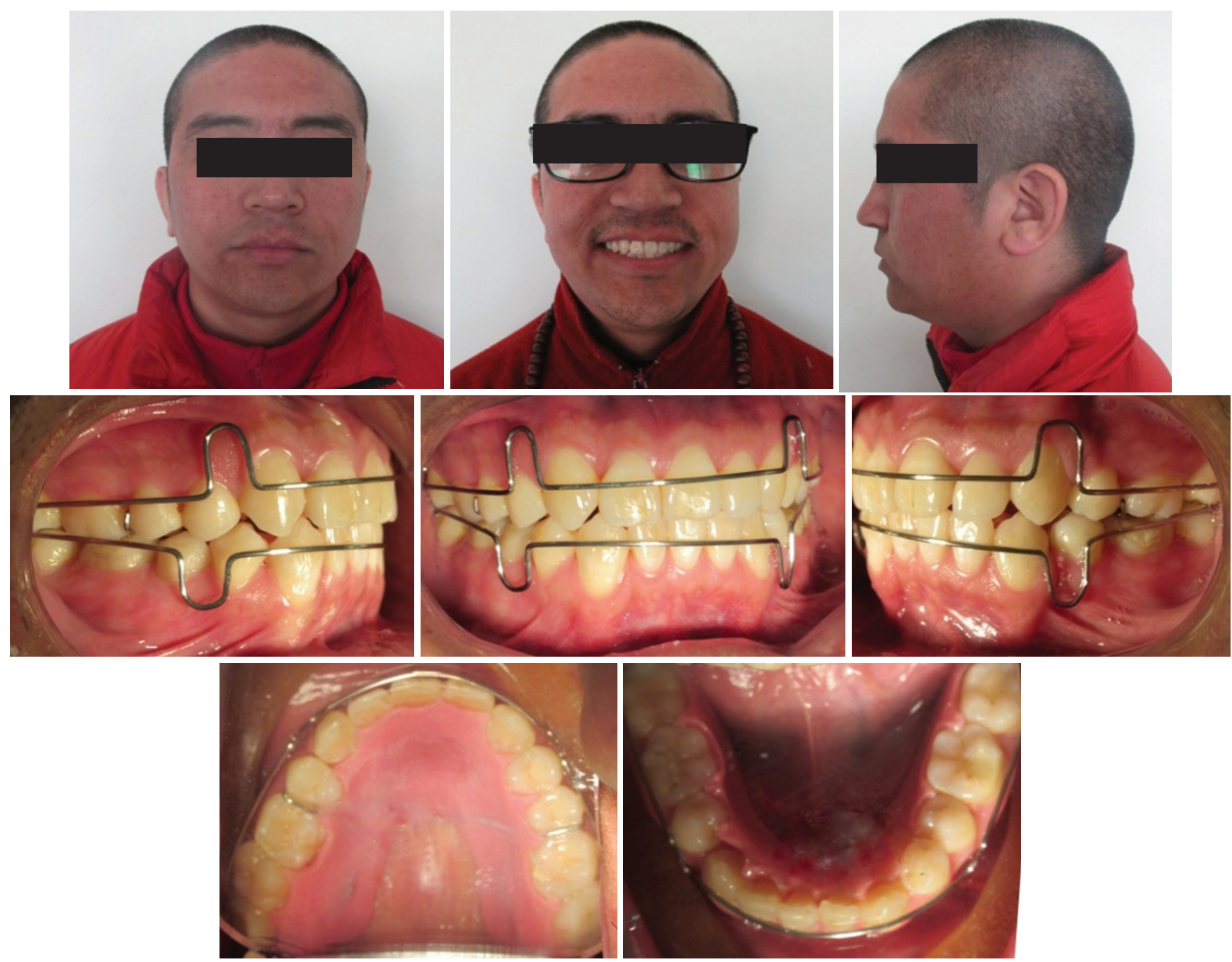

Figure 5: Post-debonding photographs with retainers

\section{RESULT}

The facial analysis showed favorable profile. Adequate incisal display with smile symmetry was achieved, corridor space was also reduced. Well aligned Class I occlusion with proper overjet and overbite were achieved with good interdigitation.
Table 1 depicłs favorable comparative cephalometric changes. OPG showed no significant root resorption or alveolar bone loss. Dental midline shift and positive lip step was corrected. Cephalographic superimposition showed upward movement of occlusal plane causing reduction of mandibular plane angle and lowered anterior face height (Figure 6).
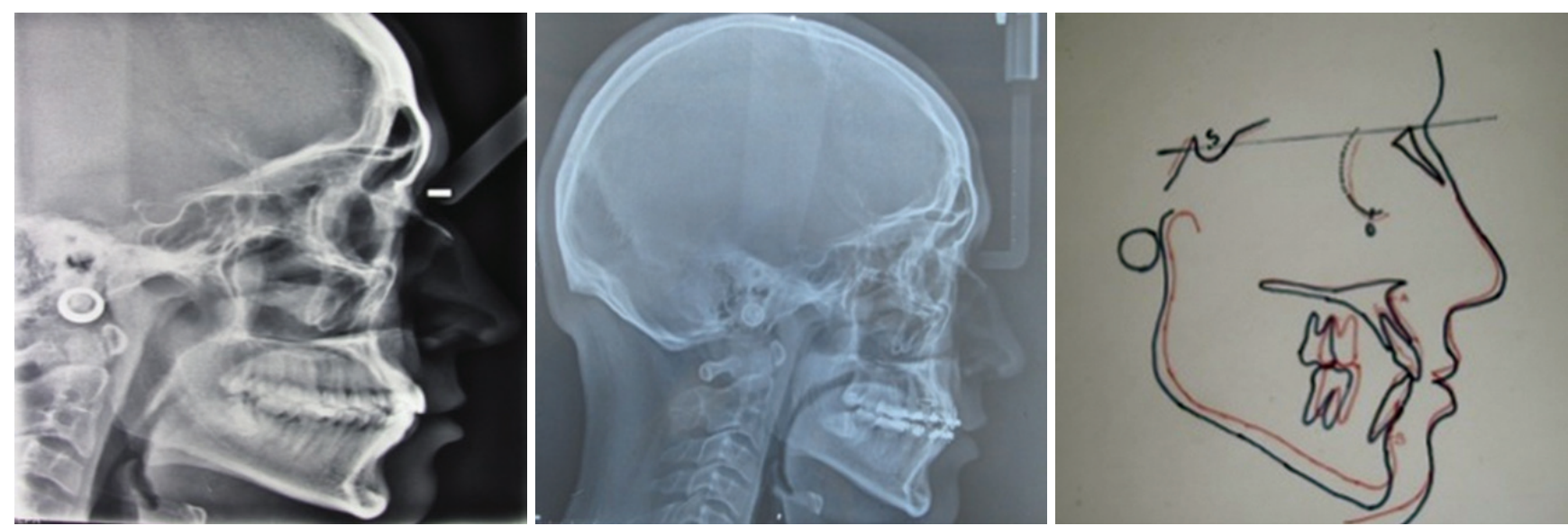

Figure 6: Pre-treatment, Post-treatment lateral cephalogram and superimposition 
Table 1: Comparison of pre-treatment and post-treatment cephalometric values

\begin{tabular}{|c|c|c|c|}
\hline Parameter & Pre-treatment & Post-treatment & Difference \\
\hline ANB (SNA, SNB) & $-2^{\circ}\left(76^{\circ}, 78^{\circ}\right)$ & $-2^{\circ}\left(76^{\circ}, 78^{\circ}\right)$ & $0(0,0)$ \\
\hline Mandibular plane angle (Steiner, Down, Tweed) & $34^{\circ}, 32^{\circ}, 33^{\circ}$ & $32^{\circ}, 28^{\circ}, 29^{\circ}$ & $-2^{\circ},-4^{\circ},-4^{\circ}$ \\
\hline Facial angle & $83^{\circ}$ & $83^{\circ}$ & 0 \\
\hline Angle of convexity & $-6^{\circ}$ & $-7^{\circ}$ & $-1^{\circ}$ \\
\hline Y-axis & $66^{\circ}$ & $66^{\circ}$ & 0 \\
\hline Maxillo-mandibular difference & $40 \mathrm{~mm}$ & $36 \mathrm{~mm}$ & $-4 \mathrm{~mm}$ \\
\hline Facial axis & $+2^{\circ}$ & $+2^{\circ}$ & 0 \\
\hline Inter-incisal angle & $110^{\circ}$ & $124^{\circ}$ & $14^{\circ}$ \\
\hline UI to $N A(L, A)$ & $39^{\circ}, 13 \mathrm{~mm}$ & $32^{\circ}, 11 \mathrm{~mm}$ & $-7^{\circ},-2 m m$ \\
\hline LI to NB $(L, A)$ & $32^{\circ}, 9 \mathrm{~mm}$ & $26^{\circ}, 7 \mathrm{~mm}$ & $-6^{\circ},-2 \mathrm{~mm}$ \\
\hline Ul to A-Pog & $9 \mathrm{~mm}$ & $7 \mathrm{~mm}$ & $-2 m m$ \\
\hline IMPA & $100^{\circ}$ & $95^{\circ}$ & $-5^{\circ}$ \\
\hline FMIA & $47^{\circ}$ & $56^{\circ}$ & $-9^{\circ}$ \\
\hline FMA & $33^{\circ}$ & $29^{\circ}$ & $-4^{\circ}$ \\
\hline S-line & $0,+2 \mathrm{~mm}$ & $0,+1 \mathrm{~mm}$ & $0,-1 \mathrm{~mm}$ \\
\hline E-line & $-5 \mathrm{~mm}, 0$ & $-4 m m,-2 m m$ & $+1 \mathrm{~mm},-2 \mathrm{~mm}$ \\
\hline Lip strain & $7 \mathrm{~mm}(18 \mathrm{~mm}, 11 \mathrm{~mm})$ & $6 \mathrm{~mm}(19 \mathrm{~mm}, 13 \mathrm{~mm})$ & $-1 \mathrm{~mm}(1 \mathrm{~mm}, 2 \mathrm{~mm})$ \\
\hline $\mathrm{H}$-angle & $20^{\circ}$ & $18^{\circ}$ & $-2^{\circ}$ \\
\hline Skeletal profile convexity & $-3 m m$ & $-4 m m$ & $-1 \mathrm{~mm}$ \\
\hline
\end{tabular}

\section{DISCUSSION}

Treatment results with one mandibular incisor extraction have been supported by case reports of Owen, ${ }^{4}$ Valinoti, ${ }^{5}$ Kokich. ${ }^{9}$ However, studies by Riedel ${ }^{10}$ suggested treatment with lower incisor extraction in certain malocclusions only. Single incisor extraction enable the clinician to produce enhanced functional and cosmetic results with minimal orthodontic manipulation.? Stability of the results with mandibular incisor extraction seemed to be better than the treatment with premolar extraction. ${ }^{10}$ Mandibular incisor extraction has some advantages such as permitting maintenance of a harmonious profile by keeping anteroposterior position of lower incisor ${ }^{11,12}$ and reducing treatment time. ${ }^{9}$ In patients with mandibular incisor crowding, both extraction and non-extraction can result in the increase of intercanine width. Extraction of a mandibular incisor reduces dental crowding without expanding the intercanine width and improves mandibular anterior root parallelism with the reduction in root proximity. It also increases the thickness of interdental bone. With this approach, posterior occlusion is not affected where interdigitation is good.

\section{REFERENCES}

1. Tweed C. A philosophy of Orthodontic treatment. Am J Orthod. 1945;31 (2):74-113.

2. Tayer B. The asymmetric extraction decision. Angle Orthod. 1992;62(4):291-7.

3. Hulsey C. An esthetic evaluation of lip teeth of lip teeth relationships present in the smile. Am J Orthod. 1970;57(2):132-44.

4. Owen A. Single lower incisor extraction. J cli Orthod. 1993;27:153-60.

5. Valinoti J. Mandibular incisor extraction therapy. Am J Orthod Dentofac Orthop. 1994;105:107-16.

6. Hunter J. Of the irregularity of the teeth. Palmer JF. 1835;12:88-92.

7. Hahn J. Problems in treatment of malocclusion. Angle Orthod. 1942;12:61-82.

8. Bolton W. Disharmony in tooth size and its relation to the analysis and treatment of malocclusion. Angle Orthod. 1958;105:107-16.

9. Kokich V, Shapiro P. Lower incisor extraction in orthodontic treatment. Angle Orthod. 1984;54:139-53.

10. Riedel R, Little R, Bui T. Mandibular incisor extraction treatment-post treatment evaluation of stability and relapse. Angle Orthod. 1992;62:113-6.

11. Uribe F, Nanda R. Considerations in mandibular incisor extraction cases. J Clin Orthod. 2009;43:45-51.

12. Canut J. Mandibular incisor exraction : indications and long term evaluation. Eur J Orthod. 1996;18:485-9. 